\title{
Tourism, Peer Production, and Location-Based Service Design
}

\author{
Eric C. Kansa \\ Information and Service Design Program \\ School of Information, UC Berkeley \\ ekansa@ischool.berkeley.edu
}

\author{
Erik Wilde \\ Information and Service Design Program \\ School of Information, UC Berkeley \\ dret@berkeley.edu
}

\begin{abstract}
This paper describes characteristics of information and service design by exploring the needs and motivations of tourists. Tourists are expected to be important and demanding users of location-based services. They will need customized means to filter their experience of destinations, as well as ways to meaningfully participate in the creation of narratives and histories about different places. Mobile technologies will also allow tourists to be more discriminating in their patronage of different service offerings, especially as they gain greater knowledge of so-called "backstage" processes. These demanding needs will require choreography between services offered by many different commercial, cultural, educational, and community providers. The paper suggests approaches to deliver tourist location-based services based on low barrier of entry principles of web architecture. The paper concludes with a discussion on how the erosion of backstage/frontstage distinctions in service systems impacts service innovation.
\end{abstract}

\section{Introduction}

Travel and mobility is one of the most striking features of contemporary society and economy. Estimates indicate some 842 million tourist arrivals in 2006 alone ${ }^{1}$. These travelers are significant consumers of services, including information services. This population is growing and increasingly demanding user community support for emerging location-based information services.

Information technologies underlie much of this growing emphasis on enhancing the delivery of "experiences." These technologies work to coordinate both in background or backstage business processes, and they enhance front-stage experiences that the consumer

\footnotetext{
${ }^{1}$ Transportation Industry Association

$<$ http://www.tia.org/researchpubs/itnl_tourism_world.html>
}

actually perceives [front-stage/backstage distinctions from Teboul 2006]. Information technologies are key drivers in the move toward mass customization that aims to satisfy individualized desires and expectations. Information technology gives firms the ability to track transactions, develop useful predictive models of customer preferences (recommendation systems and the like), and dynamically adjust price offerings (based on a multiplicity of factors, ranging from supply chain management needs to anticipated demand). Rich datastores and sophisticated data-mining and analysis enable better business intelligence and optimize decisionmaking.

While much discussion has focused on enterprise service-oriented architectures for firms involved in the tourism sector (such as airlines or hotels), we should also consider how consumers themselves draw upon an increasing variety of sophisticated information services to meet their personal needs. This paper explores the expanding penetration of information technology in tourism, particularly where those technologies empower tourists to filter and augment their own experiences. Ethnographic accounts of tourism show that tourists have under-appreciated agency in how tourist experiences are created. Pervasive and ubiquitous computing and location-based services mean tourists (and consumers in general) have more ability to discern and judge backstage processes. This shift complicates commercial efforts to stage experiences, but it also creates new opportunities for differentiation in the marketplace. Increasing access to backstage processes opens new doors for service innovation, including the possibility of firms leveraging user participation to enhance the co-production of frontstage service interfaces. Thus, many of the issues central to the study of "service science" [Spohrer et al 2007] can be explored with a closer look at how information technologies mediate tourist experiences.

Central requirements for these services will be capabilities to empower tourists to filter and augment their own experiences. Expanding mobile computing will 
mean tourists (and other consumers) will have more ability to understand and co-create their experiences. This discussion concludes with strategies to shape location-aware services to meet the needs of tourists.

\section{Tourism and Location-based Services}

Local, domestic, and international tourism all have different characteristics, participants, and underlying motivations [Gottlieb 1982; Collins et al 2002; Cole 2007]. Some of the motivations emphasize the way travel experiences help reinforce select social relationships that may be strained and attenuated by work obligations. For example, a family vacation often serves as a means of reinforcing family ties through the shared experience of travel. In other cases, travel and tourism may be related to performance and identity. One travels to certain places and in certain modes to emphasize, enact, and demonstrate desired personal characteristics. A rugged trek through the Andes can provide the experience of spectacular landscapes while demonstrating toughness and an adventurous spirit. Such travel also serves to differentiate oneself from the "mass-tourist" who travels only in comfortable and safe places while shying away from more authentic experiences.

Because tourists vary in motivations, self-perceptions, and in how they want to cast themselves, tourist services have shifted towards more diverse offerings. The growing diversity of tourism offerings stems from increasing disposable incomes and the ability of information technologies to efficiently connect niche demand with niche supply [Anderson 2006; Lew 2006]. The growing capabilities of mobile communications and locationaware services will accelerate these trends towards ever more individualized touristic experiences. Computation is rapidly becoming more mobile and ubiquitous [Greenfield 2006:48-65] and this shift has important implications for how we travel and experience places. At its heart, ubiquitous computing will enable nothing less than the customization of place. Locations will become dynamically responsive to the needs, expectations, and desires of their visitors in two primary ways:

(1) Experience Filtering: Location-based services can help tourists achieve these goals by enabling tourists to avoid experiences that distract or diverge from their goals. More and more of one's experiences of travel will be in tune with individualized goals and expectations.

(2) Experience Augmentation: Location-based services can make tourism activities more memorable, informative, and entertaining by enriching the environment with additional information. Such augmented reality technologies can enhance and transform the tourist experience of place.
In all, location-based services and ubiquitous computing will highlight and enhance desired aspects of a place while other aspects of the environment will be avoided and filtered from attention. This is not at all a utopian vision. The processes behind filtering and accentuating different aspects of reality and fantasy are inherently political, and imply significant privacy threats. The increasing power to stage places and to generate experiences that cater to individual expectations has disturbing implications. However, as will be discussed, there are powerful commercial drivers that push the types of developments explored in this paper.

\section{Recommendation Services}

Travelers already use a variety of experience filtering strategies to select and navigate destinations. Guide books, travel agents, concierge services, websites, wordof-mouth recommendations all help tourists optimize their travel experiences. Increasingly, they draw upon mobile devices. Precise navigation and geo-location combined with geo-referenced information on the Web promise to help transform travel experiences. Such situational awareness will help tourists to avoid unwanted experiences. Recommendation services and services that facilitate social exchanges between travelers have been available in the tourism sector for years [Hwang et al 2006]. Travel recommendations will become a much more pervasive experience, based on richer and more comprehensive data about individuals. Recommendations (generated socially or algorithmically) can be delivered with mapping services, in real-time on mobile devices [Hinze and Buchanan 2006]. Thus, location-based recommendation services (hereafter LBR-services) will become an increasing aspect of travel and tourism.

The privacy implications of tying recommendation services with location-based services are profound. The movements, visits, and purchase decisions of travelers will be increasingly tracked, recorded, and used to update recommendation systems. Such privacy and civil liberties concerns see different legislative outcomes between the US, the European Union, Japanese and other jurisdictions. Nevertheless, some assurance of anonymity (backed by law or not) can be important in cases where exposure of one's visit to a place carries social risks. Travel has aspects of social performance, and visiting certain places (casinos, red-light districts, etc.) can be damaging to one's reputation. While high privacy may be the ideal default setting, it makes recommendation and other services harder to deliver. Thus, there is a fundamental trade-off between service quality and privacy.

Some evidence suggests that consumers will regard recognized brands associated with over-all good customer services with good privacy protections [Ponemon Institute and TRUSTe 2008:4]. Thus, in response to 
security and privacy concerns, travelers will likely seek to use known and trusted recommendation services. These LBR-services themselves need to aggregate and filter content from a number of resources in order to better suggest destinations and experiences based on different user profiles. Aggregation will be favored because travelers may believe that privacy will be better safeguarded by using a limited set of known and trusted service providers than by sharing potentially sensitive information with many smaller service providers that have not developed reputations for trust. Thus, the need for aggregation and importance of trust in LBR-services will probably also favor large, known service providers. Information architectures that may facilitate aggregation of spatially referenced data will be discussed below.

In addition to privacy concerns, other factors will favor consolidation of LBR-services toward established Web giants. Firms that have access to more comprehensive profiles of the preferences of each individual traveler will be able to deliver more effective and mass-customized LBR-services. This will tend to favor firms that already "know" individuals and how they make choices in their day-to-day lives outside of travel. Thus, there will likely be consolidation in travel recommendation services, or at least an active trade and exchange in data about individual preferences (data about one's personal preferences can be exchanged like credit ratings). People already tend to gravitate toward information hubs, including major search engines when they plan travel [Pan and Fesenmaier 2006:825]. Google and Amazon can and do collect vast amounts of data about how individuals make choices, including travel related choices. It is very likely that these data would be very useful for LBR-services. These two Internet giants also have the computing capability and infrastructure needed to deliver recommendations to a traveler on demand. An individual tourist may either interact with Google, Amazon, or a similar firm directly, or they these giants may support backstage services for smaller actors (individual hotels, restaurants, etc.). Either way, there seems to be some tendency toward consolidation of recommendation services within a few major firms. Regardless of its "front-stage" or "backstage" manifestations, this infrastructure will enable most service providers a traveler encounters to automatically know individualized preferences and use that understanding to recommend appropriate services and experiences.

\subsection{Ubiquitous Recommendation Architectures}

In the above discussion, we suggested that aggregating spatially-referenced content can help mitigate some privacy concerns. We envision a scenario where a digital representation of travelers' tastes and desires will follow them wherever they go. If one can be automatically identified, and personal identity can be correlated with databases that document individual desires and tastes, advertising can be easily personally targeted. Advertisements for offerings that do not meet personal tastes will be filtered away. This means the experience of travel will become more and more like a visit to Amazon.com, and advertising becomes more of a helpful service than an annoying distraction. Such systems will likely become more pervasive and be used by an expanding array of travel service providers. For instance, food preferences are often as linked to selfimage as preferences for tourist destinations [O'Mahony and Hall 2007], and such preferences can also be represented in recommendation systems. When visiting a pub in a distant city, a LBR-service may inform the bartender about personal tastes even before stepping up to the counter. While this kind of ubiquitous information may prevent some unwanted experiences, it runs the risk of overly catering to tastes and expectations, possibly taking some of the serendipity and adventure out of travel.

LBR-services will be most effective and deliver the most value to users if they can draw upon, evaluate, and rank a "long tail" of content and niche service offerings provided by many different actors. A key element to making the web support ubiquitous LBR-services is to lower barriers to the exchange and aggregation of spatially referenced content. Web-based services that carry location information need to be simple enough for the participation of many different actors. In order to achieve this vision, standards development for the Web needs to better represent spatially-referenced information and transactions. Proposals for lowing barriers to location-based services are discussed below.

\section{Augmenting Travel Realities}

Location-based services will do more than filter away places a visitor has no interest in seeing. These services will also play an increasing role in enhancing the enjoyment of places by making them more immersive and captivating. Many visitors to a location like the Grand Canyon are awed by the natural spectacle of the environment. However, a variety of information resources and services (visitor centers, guides, guide books, tours, etc.) enhance the natural experience of visiting to these places. Such services enable visitors to better appreciate and understand dramatic landscapes and histories about places. These information resources and services are also highly differentiated and cater to different needs and tastes, and thus play a role in the self-expression of tourists.

Museums, civic organizations, and other traditional players now find themselves increasingly in conversation with individual tourists with regard to how places are staged and portrayed [Pan and Fesenmaier 2006:826827]. Cultural organizations, such as those that maintain 
museums, theaters, and historical sites see strategic advantage in cultivated communities engaged with their offerings. Amsterdam has an innovative service, the KPN Mobile Museum Guide, to deliver news and updates about its museums to visitors and residents. The guide includes interactive maps, news updates (coming from a weblog), tours, and community tagging of places. Similarly, the MIT Museum Without Walls Project is attempting to deliver customizable guided tours of their campus, with content delivered via location-aware technologies.

Many such cultural organizations are experimenting with integrating their own online presence with popular online community services, tools and platforms such as Facebook, Flickr and the Wikipedia. For example, the East Lothian Museum has put up a large photo set of its collections into Flickr. At the same time, RFID tags and other wireless technologies along with increasingly inexpensive display technologies are making it feasible to have museum display labels and other associated media dynamically delivered to visitors. For example, the Live!Label project aims to make labels describing museum collections dynamic through networked LCD displays [Parry et al 2007]. Potentially, a museum could deliver customized presentation of its collections for each visitor, combining both museum-developed information and information exchanged by other visitors in social media systems.

Thus, Web 2.0 type systems that invite user participation make the production of information about places increasingly decentralized. Flickr is well known for hosting millions of travel related images and making these available through geographic services. Socialight also enhances the social experience of place by allowing travelers to leave virtual notes to one another that are accessible at specific locations. Google's recently announced Open Social API allows coordinate based positions to represent the locations of people. Presumably services based on this API can further expand the social experience of places. These social software systems can enhance the social and emotional motivations for tourism by allowing tourists to connect and share experiences, play, and share emotional reactions with one another [Bruner 2005: 165-166]. However, as will be discussed below, the proliferation of such services raises important usability issues.

\subsection{Seeing Backstage}

Like media in other sectors, tourism related media is moving away from unidirectional communication toward increasingly open and participatory kinds of communication. The Web gives travelers more power to research, interpret, share, and shape their own experience as well as the experiences of their fellow travelers. Immediate access to information facilitates self- fashioning in other ways. Some tourists may feel that their experience is more meaningful if they gain some privileged insights or knowledge about a locale. As Bruner [2005:95-100] notes, not every tourist is inclined simply to accept a tourist offering at face value. Many have a "questioning gaze” and are skeptical and critical of their position both as tourists and how tour operators attempt to frame their experiences [Goodman 2007:9]. Interested tourists increasingly have access to the histories of every place, performance, scene, and cuisine that they encounter.

Thus, tour operators will have less control over how destinations are staged and portrayed [Pan and Fesenmaier 2006:826-827]. While not every tourist will be interested in digging deeply into a tourist attraction, some will and will want to share their observations. Location-based services, especially if aggregated, can make such observations easy to discover by other travelers. This has important implications for providers of tourist services. Processes and business practices that formerly happened backstage and behind the scenes will be far more likely to receive scrutiny. For example, several surveys show several studies that consumers would prefer "green" products and services and prefer to avoid products made under poor working conditions [see review: O’Rourke 2005]. Lack of information at the point of sale currently limits the impact of such preferences on consumer behavior. However, some location-based services, even a simple geographically referenced news RSS feed, may make it easy for travelers to be alerted about a hotel's labor disputes, health, and environmental records. Problems with these backstage processes will very likely hurt sales. Thus, pervasive computing and location-based services will place behind the scenes business processes under far more scrutiny.

\subsection{Peer Production of Tourist Realities}

The full potential of thee social and collaborative systems to enhance tourism experiences has yet to be reached. "Peer production", or the collaboration of volunteer communities, is emerging as a major economic force impacting the software industry, journalism, the arts, education, and many other sectors [Benkler 2006]. The examples of Flickr and Socialight (discussed above) are early examples of peer production relating to tourism.

As ubiquitous and highly mobile computing continues to develop, the impact of peer production in tourism will become increasingly apparent. Such technologies will enable users to create and engage with user generated content much more conveniently with mobile devices while they experience tourist destinations. However digital representations of places will not likely replace tourism. Tourism is a highly embodied practice that involves walking, traveling, relaxing, eating, smelling, and all sorts of other activities with no easy virtual 
surrogate [Crouch and Desforges 2003; van der Duim 2007]. Blending the real and the virtual with "augmented reality" technologies will probably have a greater impact.

Rich information resources delivered through mobile devices can help tourists learn more about the history and culture of different places. Others augmented realities may include gaming environments that transform mundane locations into places of play [see also Bruner 2005:166]. The same technologies can also transform places into fictional realities. Some augmented realities may be very elaborate fantasy environments, created by fan communities [Jenkins 2006:138-142] via peer production. For example, locations used in the filming of the Lord of the Rings movie trilogy are popular attractions for many tourists in New Zealand. Locations associated with famous authors, such as Jane Austin, are also frequented by literary tourists [Herbert 2001]. Technologies can make such tours of fictionalized landscapes and places even more immersive and memorable.

In order to enrich and enhance the experience of a augmented reality, the reality must be as richly textured and immersive as possible [see Jenkins 2006:138-142 for discussion of how fans elaborate on fictional worlds]. Keeping interactions "on theme" is a major element of successful staging of experiences [Pine and Gilmore 1999:11-15]. Machine-to-machine communications, in the form of Service Oriented Architectures (SOA) and web services can be leveraged for augmented reality applications. Web services are vital aspects of communications infrastructure that help facilitate commercial transactions and facilitate the integration of various information resources on the Web. They can be and are used to create customized interfaces to interact with common commercial services. For example, airline reservation systems can be accessed through a specific airline's web-portal or through the website of an online travel agency. The style, branding, and interface features may differ in these different portals, but they may all use common "backstage" information systems to process your credit card payment and book your reservation and create your seat assignments.

Peer production can have an important role in shaping how people interact with such backstage information systems. User communities have the potential to develop different customized interfaces for backstage web services. These interfaces can be made to fit the specific metaphors and settings of their favorite augmented reality. Each consensual reality will be able to package otherwise mundane services in metaphors appropriate to the fantasy environment.

This discussion describes some important routes toward mass-customization of tourist services. By exposing some backstage processes as web services to the public, a firm can enable user communities to develop different sorts of front-stage interfaces (see discussion below). In other words, a backstage made open and accessible to community development can facilitate peerproduction of a wide variety of service experiences.

\subsection{Architectures for Peer Producing Places}

While peer production has great potential to transform tourist experiences, there remain important barriers. Obviously, for security and privacy reasons, not every backstage business process system should be exposed to public development. Complexity is also an important impediment in engaging with community development of services.

Without aggregation strategies, user communities and user created content will be scattered across a number of silos and commercial services. A lack of aggregation makes user experience more complex and cumbersome, as users typically have to go from one travel related service to another. These hurdles unfortunately make location-based services less compelling. They currently require too much time and attention to use and often distract from the experience of places more than they enhance the experience of place.

In addition, many commercial actors each have their own custom APIs and manage their data in a mostly closed way. Such custom APIs increase the cost and difficulty of creating mashups that combine diverse webbased services. They also increase risks for developers, since they are subject to unexpected changes. A lack of open and agreed upon standards for location-based services therefore makes aggregation difficult. For communities attempting to create compelling augmented realities that draw upon a variety of location-based services and various other types of services (such as banking or transportation), open standards are essential.

\section{Consideration of General Architectures}

Tourism and leisure travel will become an important application area for augmented reality and mobile computing. However, as discussed, commercial APIs are not ideal solutions for cultivating peer production in the tourism sector. Barriers to participation and to the open accessibility of geo-referenced data need to be lowered. Simple, common standards that adhere to the architectural principles of the Web encourage participation by lowering costs and risks for developers.

Aggregation of spatially referenced content from multiple silos is an essential cornerstone for enhancing tourist experiences. Though there are many web-based applications that manage location data, the web itself currently is location-agnostic [Wilde and Kofahl 2008]. However, this situation is beginning to change. One key building block to a location-aware web is the Atom syndication format [Nottingham and Sayre 2005]. Atom 
is a reformulation of the various competing versions of the earlier RSS formats. It supports an easy way to publish any kind of self-contained information item in the form of XML. As of now, however, Atom itself has no location support. Nevertheless, GeoRSS has been developed to embed location data into RSS and Atom. This informal standard can thus be employed to create feeds of location-based data. Unlike common approaches typically deployed in enterprise settings, Atom and GeoRSS are simple to use and supporting technologies are widely deployed across the web. Thus, they can readily support large communities needed to cultivate peer production.

Atom feeds have an additional advantage in that they lend themselves to easy aggregation. As discussed above, aggregation is a crucial element for many types of location-based services important for tourism, including LBR-services and the delivery of compelling augmented realities. However, aggregation is not sufficient for the demanding needs of tourism. Standardized ways of querying a location-enabled Atom feed for entries about a certain location or area are also required. For instance, spatial querying will be required for travelers to easily learn about places they are visiting. They will need to query a variety of Atom feeds that may include images about a place, related reviews and news items, and even assessments of backstage processes generated by NGOs or environmental watchdog agencies.

Peer production requires that users not only have easy ways to retrieve information, they need to have easy ways to contribute. In addition to the Atom feed format, the Atom Publishing Protocol (AtomPub) [Gregorio and de Hóra 2007] extends the interactions with a feed to also include the submission of new entries to a feed. AtomPub itself does not make any assumptions about the submitted entry, so location-based information could be submitted using the GeoRSS format. While GeoRSS is sufficient for many purposes, future augmented reality applications will require handling of richer spatial information. Standards development in this area is only in its infancy.

Some mobile devices are already equipped with features that enable open interaction between travelers and travel-related services. For example, Nokia's Lifeblog support on some of its devices is in essence the implementation of an AtomPub client (accompanied by supporting software and services), so that travelers can submit information directly from their mobile phones to any service supporting AtomPub (many blogging services support AtomPub). Unfortunately, the current version of Lifeblog does not support location information, again illustrating how the Web standards regarding locationbased information need to mature.

The technologies discussed here have a greater potential to encourage the development of peer production systems than do other approaches toward web services. Many SOA architectures based on the WSfamily of formats are too complex for wide adoption. To best encourage peer production, we advocate the development and use of standardized protocols to support a flexible and loosely coupled ecosystem of locationbased aggregation and filtering services. Our current research work combines the above principles of locationaware information sharing, and this LocAtomPub approach has the following main characteristics:

- Open Standards: Atom and AtomPub are the two main technologies and they allow the reuse of existing tools and code.

- Geolocation: Atom is extended to support location information, which can be either geolocation (lat/long coordinates) or place names.

- Spatial Queries: Based on the location information of entries, there is a standardized way for retrieving entries only from a given area.

- Location-oriented Submission: For uploading entries through AtomPub, there is a standardized way of how location information is encoded, so that it can be recognized on the server side.

This approach combines existing pieces of the web technology landscape, rather than inventing something entirely new [Wilde 2008]. Our main goal is to make it as easy as possible for information consumers and providers to participate. A flourishing ecosystem of location-based services would be a better starting point for exploring future applications, such as augmented reality services, that will require greater spatial referencing than offered by simple latitude and longitude coordinates.

What this rather technical consideration does not address is the question of economic incentives. The current silo-based approach has clear economic incentives in forcing users to return to a particular service if they want to use their data. In the open approach, this is not necessarily true anymore. However, providers of aggregation services, especially firms that offer recommendation services, could benefit greatly since the above approaches will lower aggregation costs. They may encourage others to adopt open standards. Commercial pressures may drive many to adopt open standards so they can gain visibility in aggregation and recommendation services. Such pressures are currently most clearly seen in "Search Engine Optimization" practices including the widespread adoption of the Sitemap standard. Nevertheless, the technical, conceptual and incentive questions surrounding the cultivation of a thriving ecology of location-based services are important topics of continued research.

\section{Conclusions}

This discussion of technological and mobility trends in tourism can help illuminate common themes in other service areas. Service science aims to provide an overall 
conceptual framework for understanding how service systems develop and function, and how they can be optimized. One of the key areas of consideration in this literature centers on the front-stage/backstage distinction [Glushko and Tabas 2007; Teboul 2006]. Front-stage processes are visible to service consumers, while backstage processes happen behind the scenes.

While this distinction may offer useful heuristic value, it can also be limiting. The line-of-visibility that distinguishes the front-stage and the backstage need not be fixed. Customers sometimes have the motivation and inclination to perceive what is happening behind the scenes. Aggregation and LBR-services further enhance the ability of travelers select tourist offerings based on assessments of backstage processes. Mobile computing and location-based services can enhance the ability of travelers to evaluate backstage processes. These same technologies, especially web service technologies, also have the potential to let at least some consumers directly interface with backstage processes to completely reshape and recast front-stage experiences. Thus, consumers will have increasing power in how they to perceive, shape, and judge the services that they will use. In the emerging environment of ubiquitous information access and increasingly visible and open infrastructure, frontstage/backstage distinctions will become more blurred.

Similarly, distinctions between service providers and consumers can become blurred as backstage processes gain visibility. Web 2.0 systems deployed by museums and other cultural organizations have infrastructures designed to invite more community participation in how places are presented, customized, and experienced. Shifts toward service oriented architectures can enable similar, user-community developed customizations of even everyday commercial transactions (banking, transportation, accommodations) so that these transactions can also become part of a fanciful touristic experience. The ability to perceive and playfully build upon backstage systems will enable more experimentation in service design. In other words, increasing the degree of openness in backstage processes can enhance service innovation. However, caveats and cautions need to be addressed. If service quality is dependent on controlling variability in every front-stage and backstage exchange that happens in a service system [Glushko and Tabas 2007:7], opening up backstage systems to enable tinkering can introduce an element of risk. Understanding these risks and opportunities requires much more exploration.

The study of tourism reveals deep connections between the service economy and how people perceive themselves and how they portray themselves in performances to others. The panopticon of ubiquitous information access means that backstage processes can never be truly hidden. The ability to see, judge, and value backstage processes will have important implications of enterprises well outside of the tourism sector. This ubiquitous information access will likely mean that entire service systems that deliver products and services will more and more be subject to not just economical, but political, social, and even aesthetic evaluations. These multiple social and moral dimensions of value are central to a consumer's notion of self, and of the type of person they want to portray to others. Thus, we are beginning to see "green" business processes in everything from organic food to the delivery of consumer technologies. These cases, as well as this study of tourism, reveal that the quality of backstage processes can be vitally important to consumer goals in self-fashioning. They also illustrate how "front-staging" the backstage can offer important ways for businesses to differentiate their product and service offerings.

\section{References}

[1] Anderson, Chris. 2006. The Long Tail: Why the Future of Business is Selling Less of More. Hyperion.

[2] Benkler, Yochai. 2006. The Wealth of Networks: How Social Production Transforms Markets and Freedom. Yale University Press.

[3] Bruner, Edward M. 2004. Culture on Tour: Ethnographies of Travel. University Of Chicago Press.

[4] Cole, Stroma. 2007. "Beyond authenticity and commodification." Annals of Tourism Research 34(4):943-960.

[5] Collins, Darrian, and Trisdell. 2002. "Age-related Lifecycles: Purpose Variations.” Annals of Tourism Research 29(3):801-818.

[6] Crouch, David, and Luke Desforges. 2003. "The Sensuous in the Tourist Encounter: Introduction: The Power of the Body in Tourist Studies.” Tourist Studies 3(1):5-22.

[7] Glushko, Robert J., Lindsay Tabas. 2007. "Bridging the 'Front Stage' and 'Back Stage' in Service System Design”. (June 15, 2007). School of Information. Technical Paper 2007-013 <http://repositories.cdlib.org/ischool/2007-013>

[8] Goodman, Elizabeth. 2007. "Destination Services: Tourist media and networked places" (March 2, 2007). UC Berkeley, School of Information. Technical Report No. 2007-004.

[9] Gottlieb, Alma. 1982. “Americans' vacations.” Annals of Tourism Research 9(2):165-187. 
[10] Gray, Noella J., and Lisa M. Campbell. 2007. “A Decommodified Experience? Exploring Aesthetic, Economic and Ethical Values for Volunteer Ecotourism in Costa Rica." Journal of Sustainable Tourism 15(5):463-482.

[11] Greenfield, Adam. 2006. Everyware: The Dawning Age of Ubiquitous Computing. 1st ed. New Riders Publishing.

[12] Gregorio, Joe and Bill de Hóra. "The Atom Publishing Protocol.” Internet RFC 5032, October 2007.

[13] Haghighat, Amir, Cristina Videira Lopes, Tony Givargis, and Atri Mandal. "Location-Aware Web System." In Proceedings of the Workshop on Building Software for Pervasive Computing at OOPSLA'04, Vancouver, Canada, October 2004.

[14] Herbert, David. 2001. "Literary places, tourism and the heritage experience." Annals of Tourism Research 28(2):312-333.

[15] Hinze, Annika, and George Buchanan. 2006. “The challenge of creating cooperating mobile services: experiences and lessons learned." Proceedings of the 29th Australasian Computer Science Conference, Volume 48:207-215.

[16] Hwang, Yeong-Hyeon, Ulrike Gretzel, Zheng Xiang, and Daniel R. Fesenmaier. 2006. "Information Search for Travel Decisions." In Destination Recommendation Systems: Behavioral Foundations and Applications, eds. Daniel R. Fesenmaier, Karl W. Wöber, and H. Werthner. CABI Publishing, p. 3-16.

[17] Jenkins, Henry. 2006. Fans, Bloggers, and Gamers: Media Consumers in a Digital Age. NYU Press.

[18] Lew, Alan A. 2006. "Long Tail Tourism: Implications of the distributed business model for the tourism and travel industry" ed. N. Othman. Conference Proceedings: The 2nd Tourism Outlook Conference Tourism Edge and Beyond, p. 26-38.

[19] Nottingham, Mark and Robert Sayre. "The Atom Syndication Format.” Internet RFC 4287, December 2005.

[20] Open Geospatial Consortium. "OpenGIS Geography Markup Language (GML).” OGC 06-050r3, July 2006.
Production and Consumption." Journal of Industrial Ecology 9:115-128.

[22] Pan, Bing, and Daniel R. Fesenmaier. 2006. "Online Information Search: Vacation Planning Process.” Annals of Tourism Research 33(3):809-832.

[23] Parry, Ross, Mayra Ortiz-Williams, and Andrew Sawyer. 2007. "How Shall We Label Our Exhibit Today? Applying the Principles of On-Line Publishing to an OnSite Exhibition." Museums and the Web 2007. $<$ http://www.archimuse.com/mw2007/papers/parry/parry. html> [Accessed January 23, 2008].

[24] Pine, B. Joseph, and James H. Gilmore. 1999. The Experience Economy: Work Is Theater \& Every Business a Stage. 1st ed. Harvard Business School Press.

[25] Ponemon Insitute LLC, and Truste. 2008. 2007 Most Trusted Companies for Privacy Award. http://www.truste.org/pdf/2007_Most_Trusted_Companie s_Award.pdf [Accessed April 24, 2008].

[26] Snell, James. "Atom License Extension.” Internet RFC 4946, July 2007.

[27] Spohrer, Jim, Paul P. Maglio, John Bailey, Daniel Gruhl. 2007. "Steps Toward a Science of Service Systems." Computer 40(1):71-77.

[28] Stronza, Amanda. 2001. "Anthropology of Tourism: Forging New Ground for Ecotourism and Other Alternatives” Annual Review of Anthropology 30(1):261283.

[29] Teboul, James. 2006. Service Is Front Stage: Positioning Services for Value Advantage. Palgrave Macmillan.

[30] Van der Duim, Rene. 2007. "Tourismscapes an actor-network perspective." Annals of Tourism Research 34(4):961-976

[31] Wilde, Erik and Martin Kofahl. "The Locative Web". First International Workshop on Location and the Web (LocWeb 2008), pages 1-8, Beijing, China, April 2008.

[32] Wilde, Erik. "The Plain Web". First International Workshop on Understanding Web Evolution (WebEvolve2008), pages 79-83, Beijing, China, April 2008.

[21] O'Rourke, Dara. 2005. "Market Movements: Nongovernmental Strategies to Influence Global 